\title{
QUALITY OF LIFE AND ORAL PAIN IN HEAD AND NECK CANCER PATIENTS RECEIVING RADIO/CHEMORADIOTHERAPY
}

Maria Kouri ${ }^{1}$, Ourania Nicolatou-Galitis ${ }^{1}$, Athina Vadalouca ${ }^{2}$, Vasilios Kouloulias ${ }^{3}$, Georgios Maropoulos ${ }^{4}$, Panagiotis Kanellopoulos ${ }^{4}$, Efthymios Kyrodimos ${ }^{5}$, Erofili

Papadopoulou $^{1}$, Emmanouil Vardas ${ }^{1}$, Evangelos Galitis ${ }^{6}$ Miltiadis Trichas $^{7}$, Anastasia Vourli ${ }^{1}$, Anna Zigogianni ${ }^{8}$, Zoi Liakouli ${ }^{8}$, Amanda Psyrri ${ }^{9}$, Ioannis Kouvaris ${ }^{8}$

${ }^{1}$ Clinic of Hospital Dentistry, School of Dentistry, National and Kapodistiran University of Athens, Athens, Greece 2Pain Clinic, "Athens Medical Center", Athens, Greece

${ }^{3} 2^{\text {nd }}$ Radiology Department, Radiotherapy Unit, Medical School, National and Kapodistrian University of Athens, Athens Greece ${ }^{4}$ Department of Biochemistry, "Laiko" General Hospital of Athens, Athens, Greece

ELLENIC REPUBLIC UNIVERSITY OF ATHENS
${ }^{5}$ ENT Clinic "Hippokrateio" Hospital, Medical School, National and Kapodistrian University of Athens, Athens Greece

${ }^{6}$ Clinic of Orofacial Pain, School of Dentistry, National and Kapodistiran University of Athens, Athens, Greece ${ }^{7}$ Radiotherapy Department, "Iaso" Hospital, Athens, Greece

${ }^{8} 1^{\text {st }}$ Radiology Department, Radiotherapy Unit, Medical School, “Aretaieion” University Hospital, National and Kapodistrian University of Athens, Greece

90ncology Department, "Attikon" University Hospital, Medical School, National and Kapodistrian University of Athens, Athens Greece

\section{OBJECTIVES:}

To assess pain related changes on Quality of Life $(\mathrm{QoL})$, in Head/Neck cancer patients receiving radio/chemoradiotherapy.

\section{PATIENTS \& METHODS}

\section{QoL ASSESSMENT:}

Core-30 (EORTC QLQ-C30)V3.0

Head and Neck module (QLQ-H\&N35)

QoL was Assessed:

before radiotherapy $(\mathrm{t} 0), 5^{\text {th }}-7^{\text {th }}$ week $(\mathrm{t} 1)$.
ORAL PAIN EVALUATION:

Verbal Analogue Scale (VAS). Scores: $0-10$;

1 - 4: mild, 5-7: moderate,

8 - 10: severe.

\begin{tabular}{|l|r|c|c|}
\hline \multicolumn{2}{|c|}{ PARAMETERS } & N=30 & $\%$ \\
\hline \multirow{2}{*}{ Gender } & Male & 26 & 86.7 \\
\cline { 2 - 4 } & Female & 4 & 13.3 \\
\hline Age (Mean) & 64.3 & \\
\hline Radiotherapy & Postoperative & 17 & 56.7 \\
\cline { 2 - 4 } & Radical & 13 & 43.3 \\
\hline Total Dose (Mean, Gy) & 65.38 & \\
\hline Concomitant Chemotherapy & 23 & 76.7 \\
\hline \multirow{2}{*}{ Tumor Site } & Oral/ & 14 & 46.7 \\
\cline { 2 - 4 } & Oropharyngeal & & 20 \\
\cline { 2 - 4 } & Laryngeal & 6 & 10 \\
\cline { 2 - 4 } & Parotid & 3 & 23.3 \\
\hline
\end{tabular}

\section{CONCLUSIONS:}

Pain was related to most of the QoL score changes. Appropriate interventions can be directed towards managing the pain and improving QoL of the patients.

\begin{tabular}{|c|c|c|c|}
\hline \multicolumn{4}{|c|}{ PARAMETERS } \\
\hline PAIN & $\mathbf{N}$ & $\begin{array}{c}\text { QoL Values } \\
\text { Core-30 (EORTC QLQ-C3o) }\end{array}$ & $\begin{array}{c}\text { Paired } \\
\text { t-test }\end{array}$ \\
\hline \multirow[t]{2}{*}{ Severe } & \multirow[t]{2}{*}{7} & Global QoL/ Physical/ Role / Social Functions & $<0.006$ \\
\hline & & Fatigue/ Dyspnoea/ Appetite Loss & $<0.004$ \\
\hline \multirow[t]{2}{*}{ Moderate } & \multirow[t]{2}{*}{10} & Physical/ Role/ Emotional/ Cognitive/ Social Functions & $<0.017$ \\
\hline & & Fatigue/ Dyspnoea/ Appetite Loss/ Constipation & $<0.029$ \\
\hline \multirow[t]{2}{*}{ Mild } & \multirow{2}{*}{9} & Physical/ Role / Social Functions & $<0.038$ \\
\hline & & Fatigue/ Appetite Loss & $<0.039$ \\
\hline
\end{tabular}

\begin{tabular}{|c|c|c|c|}
\hline \multicolumn{4}{|c|}{ PARAMETERS } \\
\hline PAIN & $\mathbf{N}$ & $\begin{array}{c}\text { QoL Values } \\
\text { Head and Neck module (QLQ-H\&N35) }\end{array}$ & $\begin{array}{c}\text { Paired } \\
\text { t-test }\end{array}$ \\
\hline \multirow{3}{*}{ Severe } & \multirow[t]{3}{*}{7} & Swallowing/ Dry Mouth/ Opening Mouth & 0.000 \\
\hline & & Senses/ Social Eating/ Sticky Saliva & 0.001 \\
\hline & & Speech/ Social Contact/ Sexuality/ Felt Ill/ Weight Loss & $<0.030$ \\
\hline \multirow[t]{2}{*}{ Moderate } & \multirow[t]{2}{*}{10} & Swallowing/ Senses/ Social Eating/ Dry Mouth/ Sticky Saliva & $<0.007$ \\
\hline & & Social Contact/ Sexuality/ Felt Ill/ Weight Loss & $<0.029$ \\
\hline \multirow[t]{2}{*}{ Mild } & \multirow[t]{2}{*}{9} & Speech/ Social Eating/Sticky Saliva/ Weight Loss & $<0.004$ \\
\hline & & Senses/ Dry mouth/ Social Contact & $<0.035$ \\
\hline
\end{tabular}

Severe pain: Global QoL, Physical, Role, Social Functions were significantly decreased $(\mathrm{p}<0.006)$ (tl vs t0 assessment). Insignificant reduction of Emotional and Cognitive Functions; Symptom Scales and H\&N35 scores were significantly increased. Nausea/Vomiting, Insomnia, Constipation, Financial problems, Diarrhoea, Teeth problems, Coughing and Weight gain, statistically insignificant.

Moderate pain: Significantly lower Functional Scales scores $(p<0.02)$ (tl vs t0 assessment). Insignificant reduction of Global QoL was $(p=0.160)$; Symptom Scales and H\&N35 scores were significantly increased, except for Nausea/Vomiting, Insomnia, Financial problems, Diarrhoea, Speech and Teeth problems, Opening mouth, Coughing and Weight gain (statistically insignificant) 